\title{
Morphology and Growth Kinetic Advantage of Quenched Twinned Dendrites in Al-Zn Alloys
}

\begin{abstract}
MARIO A. SALGADO-ORDORICA, ANDRÉ B. PHILLION, and MICHEL RAPPAZ
Twinned dendrites appearing in an Al-26 wt pet $\mathrm{Zn}$ alloy have been quenched during growth using a specifically designed setup that is positioned on top of a directional solidification experiment. X-ray tomography performed at the Swiss Light Source (SLS-beamline TOMCAT) allowed us to reconstruct the $3 \mathrm{D}$ morphology of these structures and to confirm previous observations performed on single 2D sections (Henry et al., Metall Mater Trans A 35A:24952501, 2004; Salgado-Ordorica and Rappaz, Acta Mater 56:5708-5718, 2008). Further characterization of these quenched specimens led to a better description of the mechanisms involved in the in-plane and lateral growth propagation of twinned dendrites. These were then put into relation with the competition mechanisms taking place during simultaneous solidification of twinned and regular dendrites.
\end{abstract}

DOI: $10.1007 / \mathrm{s} 11661-012-1539-0$

(C) The Minerals, Metals \& Materials Society and ASM International 2013

\section{INTRODUCTION}

TWINNED dendrites are the base microstructural feature of feathery grains, i.e., large fan-shaped grains that sometimes occur during the semi-continuous casting of industrial aluminum alloys. Owing to their abnormal size and shape, these grains induce the formation of segregation bands that modify the mechanical and fatigue properties of the ingot and thus are considered to be defects. In previous studies, it has been shown that twinned dendrites can nucleate during solidification when a strong thermal gradient and some convection currents are present in the melt near the liquidus isotherm. ${ }^{[1-5]}$ Once nucleated, they rapidly overgrow columnar dendrites and even sometimes regular equiaxed dendrites. ${ }^{[6-8]}$ Despite numerous studies which have contributed to the understanding of such morphologies since the first observations of Herenguel, ${ }^{[1,9]}$ more than 60 years ago, many open questions remain.

Optical and electronic microscopy observations combined with EBSD and EDX measurements made over the past fifteen years on such microstructures ${ }^{[6,7,10,11]}$ have shown that (1) The primary trunks of twinned dendrites grow along $\langle 110\rangle$ directions and are split in their centers by a straight boundary that corresponds to a coherent $\{111\}$ twin plane; (2) Secondary arms grow not only along $\langle 110\rangle$ but also sometimes along $\langle 100\rangle$ directions and meet at wavy-like $\{111\}$ incoherent

MARIO A. SALGADO-ORDORICA, formerly PhD student with the Computational Materials Laboratory, Ecole Polytechnique Fédérale de Lausanne, Lausanne, Switzerland, is now Development Engineer, Novelis Switzerland, Sierre, Switzerland. Contact e-mail: mario. salgado@novelis.com ANDRÉ B. PHILLION, formerly Post-doc with the Computational Materials Laboratory, Ecole Polytechnique Fédérale de Lausanne, is now Assistant Professor, with the School of Engineering, University of British Columbia, Kelowna, BC, Canada, and MICHEL RAPPAZ, Director, is with the Computational Materials Laboratory, Ecole Polytechnique Fédérale de Lausanne.

Manuscript submitted March 25, 2012.

Article published online February 21, 2013 boundaries; (3) These growth mechanisms create an alternated sequence of twinned and untwinned lamellae, separated by a sequence of coherent/planar and incoherent/wavy twin boundaries.

Although a fairly good insight into the overall morphology of twinned dendrites has been gained thanks to these recent studies, the detailed growth mechanisms, the tip morphology, and the kinetics advantage that the twinned dendrites can have under certain circumstances over regular dendrites are still not fully clear. Henry et al. ${ }^{[10,11]}$ made a conjecture that the twinned dendrite tip is in fact a doublon, i.e., a double-tip dendrite growing with a thin liquid channel in the center, the root of which contains the twin plane that solidifies at a solid composition close to $C_{0}$. Recent phase-field simulations ${ }^{[12-14]}$ as well as FIB observations ${ }^{[15]}$ made by the present authors also strongly support this conjecture. On the other hand, if the propagation of twinned dendrites along the twin plane can be easily understood by branching mechanisms similar to those of regular columnar dendrites, their extension perpendicular to the twin plane, in particular the formation of new twins, remains unclear.

One major challenge to better understand the growth mechanisms of twinned dendrites is that their occurrence requires simultaneous melt convection and a high thermal gradient, i.e., a situation typically encountered in DC casting near the melt inlet. It is therefore difficult, if not impossible, to produce them in small crucibles under well controlled conditions and equally important, to quench or to observe them in situ during steady-state growth.*

\footnotetext{
*When trying to grow twinned dendrites from a twinned seed in a Bridgman furnace, we observed that individual regular dendrites only form. ${ }^{[16]}$ Anada et al.$^{[17]}$ could produce successfully Al-7 wt. \% Mg twinned dendrites in a Bridgman setup, thanks probably to the presence of magnesium and the large diameter of the crucibles $(25 \mathrm{~mm})$ which not only induced enough solutal convection, but also made quenching very inefficient.
} 
Considering these previous studies and the potential interest directed toward quenching of twinned dendrites during their growth, we have devised a new setup and methodology based on the DS technique. It is presented in the next section. Quenched twinned dendrites produced with this device are then characterized by X-ray tomography microscopy (1) to evaluate the effect of convection on twin propagation, and (2) to elucidate the complex branching mechanisms and twin dendrite tip shape (Section III). These results have been subdivided into three main parts: (1) 3D morphology of twinned dendrites; (2) twin propagation mechanisms; and (3) growth competition mechanisms. Finally, an updated explanation of the growth kinetics advantage of twinned dendrites is proposed.

\section{EXPERIMENTAL SET-UP}

In order to quench twinned dendrites during their growth and to possibly see their tip morphology, the DS installation used by Salgado and Rappaz, ${ }^{[7]}$ which has significant natural convection, and that has been described in detail elsewhere, ${ }^{[6,18]}$ was adapted with a quenching device placed on top of the DS mold. To summarize, the DS mold consists of a stainless steel cylinder of 55-mm ID/59-mm OD, closed at the bottom by a $0.5-\mathrm{mm}$-thick sheet. The lateral surface of the mold is tightly wrapped by a heating Kanthal wire, itself being surrounded by a layer of fiberglass wool for insulation. The internal surface of the mold is coated with sprayed $\mathrm{BN}$ particles. Cooling is initiated and controlled by a water spray underneath the base plate of the stainless steel crucible. The quenching device, also made out of stainless steel, is shown in Figure 1. The diameter of this device is reduced progressively stepwise from $35 \mathrm{~mm}$ at the base to $5 \mathrm{~mm}$ at the top. Similar to the DS mold, the quenching device is surrounded by a heating Kanthal

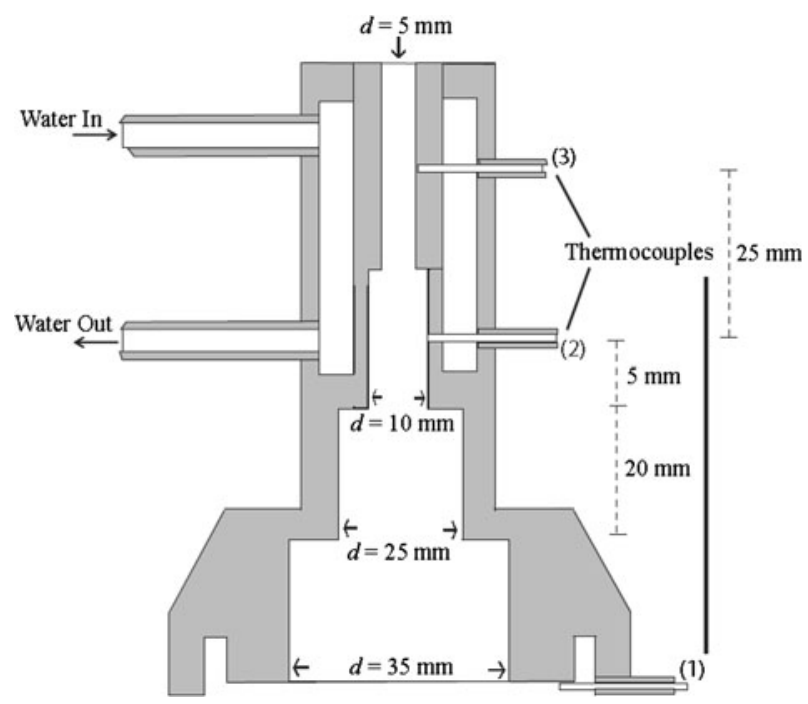

Fig. 1-Quenching device for the DS experiment. The cooling system of the thinnest region on the left side is activated and controlled by the temperatures measured by the thermocouples located on the right side. wire and a corresponding insulating layer of fiberglass wool. The quenching device contains a secondary cooling system in the thinnest section to initiate the quench when the twinned dendrites reach this location. The quenching time is controlled by the temperature measured by three thermocouples ((1),(2), and (3) as shown in Figure 1).

Experiments were conducted on an Al-26 wt pet Zn alloy, which was prepared by adding $\mathrm{Zn}$ to molten $\mathrm{Al}$ (both elements of 99.995 pct purity). A small specimen of this alloy was solidified in a graphite crucible to obtain a cooling curve and to determine the liquidus temperature $\left[T_{\text {liq }}=885 \mathrm{~K}\left(612^{\circ} \mathrm{C}\right)\right]$. After pre-heating the mold and the quenching device to $1073 \mathrm{~K}\left(800^{\circ} \mathrm{C}\right)$, using the two Kanthal heating systems, the melt was poured into it, and the water spray turned on immediately after. When the thermocouple (1) reached $T_{\text {liq }}$, i.e., when the twinned dendrite tips reached this location, the heating wire surrounding the DS mold was turned off. The secondary cooling system of the quenching device was activated only when the liquidus isotherm reached the position of thermocouple (3).

After quenching, a transverse section of the specimen was made in the $25-\mathrm{mm}$ diameter region of the quenching device. This section was mirror polished and etched to reveal the microstructure and to identify the presence of feathery grains. After verifying the presence of twins within the casting, the specimen was again polished, but then etched electrochemically to perform an EBSD analysis of the twin planes orientation. A second, longitudinal section was then obtained from the thinnest region of the casting, in the region quenched by the secondary cooling system. This section was cut parallel to $G$ and perpendicular to the twin planes. An electrochemical etching known as anodic oxidation or "Barker etching" was used to reveal the quenched microstructure, while a chemical etching was also performed with a diluted Keller solution to investigate the twin morphology.

In order to characterize in 3D the solidified morphology near the twin dendrite tip by X-ray tomography microscopy, a small specimen of $1 \mathrm{~mm}$ in diameter was extracted from the quenched region. The tomography measurements were made at the X02DA TOMCAT beamline of the Swiss Light Source (SLS), Paul Scherrer Institute, Villigen, Switzerland, using an X-ray beam energy of $20 \mathrm{keV}$ and an exposure time of 0.225 seconds for each of the 1500 projections. The image resolution was $1.48 \mu \mathrm{m}$ (binning $2 \times 2$ ) recorded with a CCD camera of $2048 \times 2048$ pixels.

\section{RESULTS AND DISCUSSION}

\section{A. Quenched Microstructure}

The twinned dendrite microstructure of an Al26wt pet $\mathrm{Zn}$ alloy produced using the quenching apparatus described in the previous section is shown in Figure 2. Figure 2(a) was obtained using the anodic oxidation etchant, while Figure 2(b) is the same longitudinal section, but after etching with a diluted Keller 


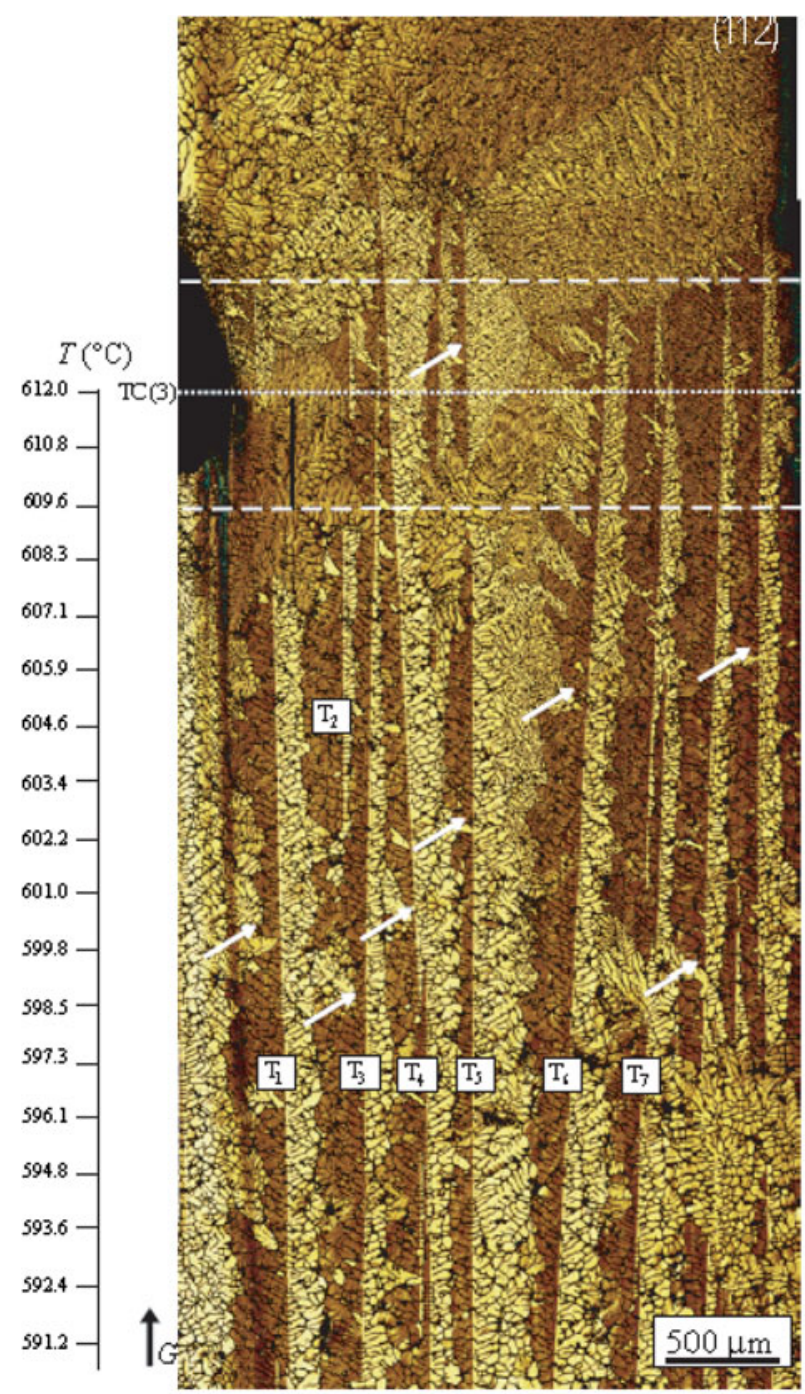

(a)

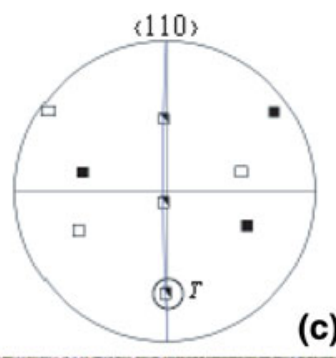

(c) (112)

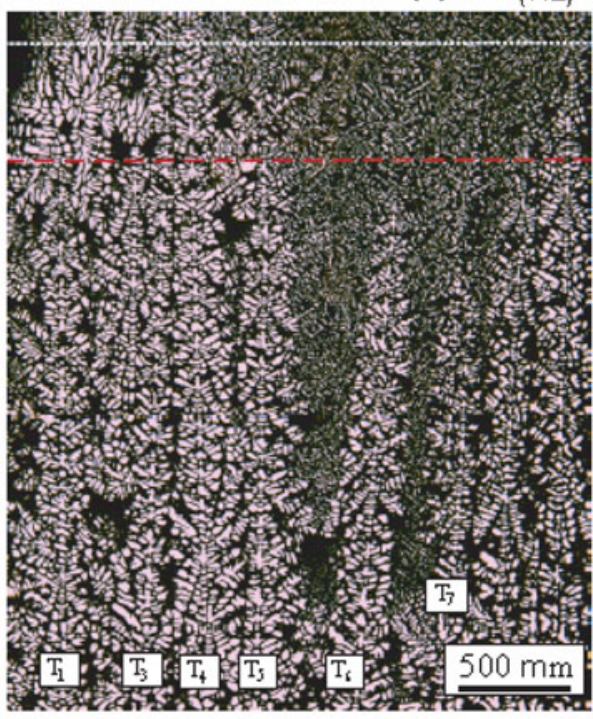

(b)

Fig. 2- Quenched microstructure of an Al-26wt pet Zn specimen produced in the DS installation of Fig. 1: $(a)$ after anodic oxidation; $(b)$ after Keller etching. In both microgrpahs, several twinned dendrites are labeled from left to right as $T_{i}$, where $i=1,2, \ldots 7$. (c) $\langle 110\rangle$ pole figure drawn from the crystallographic orientation of $T_{5}$. The location of the thermocouple controlling the temperature on the quenching device is indicated on the left. It corresponds to the liquidus isotherm (white dotted line) at the time of quench, as indicated by the temperature scale. The small white arrows identify some of the regions where the untwinned lamellae extend into the twinned regions.

solution. This longitudinal section is perpendicular to the twin plane, i.e., (112) plane. EBSD analysis showed that all the twinned (brown/dark grey) and untwinned (yellow/light grey) lamellae in Figure 2(a) correspond to a single feathery grain, although slight misorientations are present within each set of lamellae. Many of these twinned dendrites have been labeled from left to right as $T_{i}$, with $i=1,2, \ldots 7$. The $\langle 110\rangle$ pole figure in Figure 2(c), drawn from the crystallographic orientation of $T_{5}$, clearly indicates the twinned nature of these structures. As can be seen, three $\langle 110\rangle$ directions of the twinned lamella (empty squares) are in a symmetric relationship with three $\langle 110\rangle$ directions of the untwinned lamellae with respect to one common (111) twin plane (vertical arc of a circle drawn at the center of the pole figure) containing the three other common $\langle 110\rangle$ directions (partially filled squares). Therefore, we were successful in propagating the twinned structure in the narrowest part of the quenching device, where natural convection is certainly reduced.

Based on the reading of thermocouples (2) and (3) when the liquidus isotherm passes through thermocouple (2), the thermal gradient $G$ slightly ahead of the liquidus is about $9.5 \mathrm{~K} \mathrm{~cm}^{-1}$, while the liquidus isotherm velocity $v_{T} \approx 0.54 \mathrm{~mm} \mathrm{~s}^{-1}$. The mushy zone length was also determined from the temperature difference between these two thermocouples at the quenching time and was estimated to be approximately $21.2 \mathrm{~mm}$. A temperature scale is provided in Figure 2(a) and shows the temperature field in the specimen just before the quench. The liquidus $\left[885 \mathrm{~K}\left(612{ }^{\circ} \mathrm{C}\right)\right]$ was positioned at the height of thermocouple (3) and is indicated by a dotted line. This line is in the middle of two dashed lines, the spacing of which corresponds to 
the diameter $(1 \mathrm{~mm})$ of the thermocouple (the bottom white dashed line is extended in red/grey in Figure 2(b)). Although the quenched interface is not perfectly flat in this micrograph, it can be clearly seen that the transition from a twinned to a quenched equiaxed structure occurs within the region where the thermocouple is located. Near thermocouple (3), an equiaxed grain has nucleated and partially prevents further growth of the twinned dendrite trunks $T_{1}$ and $T_{2}$. The other twinned dendrites further grow during the quench, with a microstructure that is as fine as that of the equiaxed grains formed during quenching. This unfortunately prevents any observation of a quenched twinned dendrite tip shape in this specimen.

The average spacing of the twin planes measured between $T_{1}$ and $T_{7}$ at the bottom of Figure 2(a), i.e., excluding $T_{2}$, gives an average spacing of the twinned dendrite trunks $\lambda_{\perp} \approx 270 \mu \mathrm{m}$. In a previous study, ${ }^{[7]}$ it was shown that $\lambda_{\perp}$ is close to the in-plane primary spacing $\lambda_{/ /}$. Under the assumption of a square array of regular dendrites, the model of Kurz et al. ${ }^{[19]}$ predicts a primary spacing $\lambda_{1} \approx 630 \mu \mathrm{m}$, which is substantially larger than the spacing observed in Figure 2. This discrepancy can be due to (1) The morphology of secondary arms which is much more complex in the case of twinned dendrites (presence of both $\langle 100\rangle$ and $\langle 110\rangle$ arms); (2) Unsteady-state effects associated with the change of the section near the chill device; and (3) Microsegregation at the scale of primary trunks if one considers a doublon-type morphology. ${ }^{[14,15]}$

The small white arrows in Figure $2 \mathrm{a}$ indicate regions where the continuity of the coherent twin planes is interrupted by the extension of an untwinned lamellae into a twinned region. This phenomenon had already been observed in Al-Cu alloys, ${ }^{[7]}$ but not previously in an Al-Zn specimen. For some of these regions, for instance, along the lamellae on each side of $T_{1}$, it is not clear if this occurs by growth of the untwinned lamellae (yellow/light grey region) of $T_{1}$ or by that of its left neighbor into the twinned lamellae (brown/dark grey region) of $T_{1}$. However, for most cases, it is the first mechanism that is occurring. A magnified micrograph showing an untwinned extension event in the dendrite $T_{5}$ is shown in the longitudinal section of Figure 3, along with a corresponding $\langle 110\rangle$ pole figure. The twinned and untwinned lamellae appear now in dark and light gray shades, respectively. A lateral $\langle 110\rangle$ arm having the crystallographic orientation of the untwinned lamella on the right extends into the left twinned lamella. It is rather difficult to say if it grows at 60 or 90 deg.(lateral arm $L_{\mathrm{u}}^{2}$ or perpendicular $\operatorname{arm} P_{\mathrm{u}}$ in pole figure) from the trunk, given that the pole figure also indicates that the trunk is oriented about 18 deg from the observed projection. This shows that the untwinned extension event has occurred at a level of a secondary (or higher order) side branch. As will be discussed in Section III-C, these extensions might also be related to lateral twin propagation.

\section{B. 3D-Reconstruction of Twinned Dendrites}

In order to visualize the morphology and distribution of twinned dendrites in a quenched DS specimen, a cylindrical volume $1 \mathrm{~mm}$ in diameter of a portion of the microstructure shown in Figure 2 was reconstructed from the $2 \mathrm{D}$ radiographs obtained at the X-ray tomography beamline TOMCAT of the Swiss Light Source. Figure 4 shows the 2D sections of the reconstructed microstructure. They were selected by means of the visualization software Avizo 6.0. The dark and light gray areas in this reverse contrast image correspond to Zn-lean dendrites and Zn-rich interdendritic regions, respectively. The good contrast seen in this figure demonstrates the usefulness of quenching Al- $\mathrm{Zn}$ dendrites, as it strongly decreases back-diffusion in the solid state, and such 3D reconstructions clearly help in visualizing the orientation and distribution of side arms. A section parallel to a (112) plane from a region near the quenched interface is shown in Figure 4(a), along with a transverse slice (at the bottom) and a section parallel to

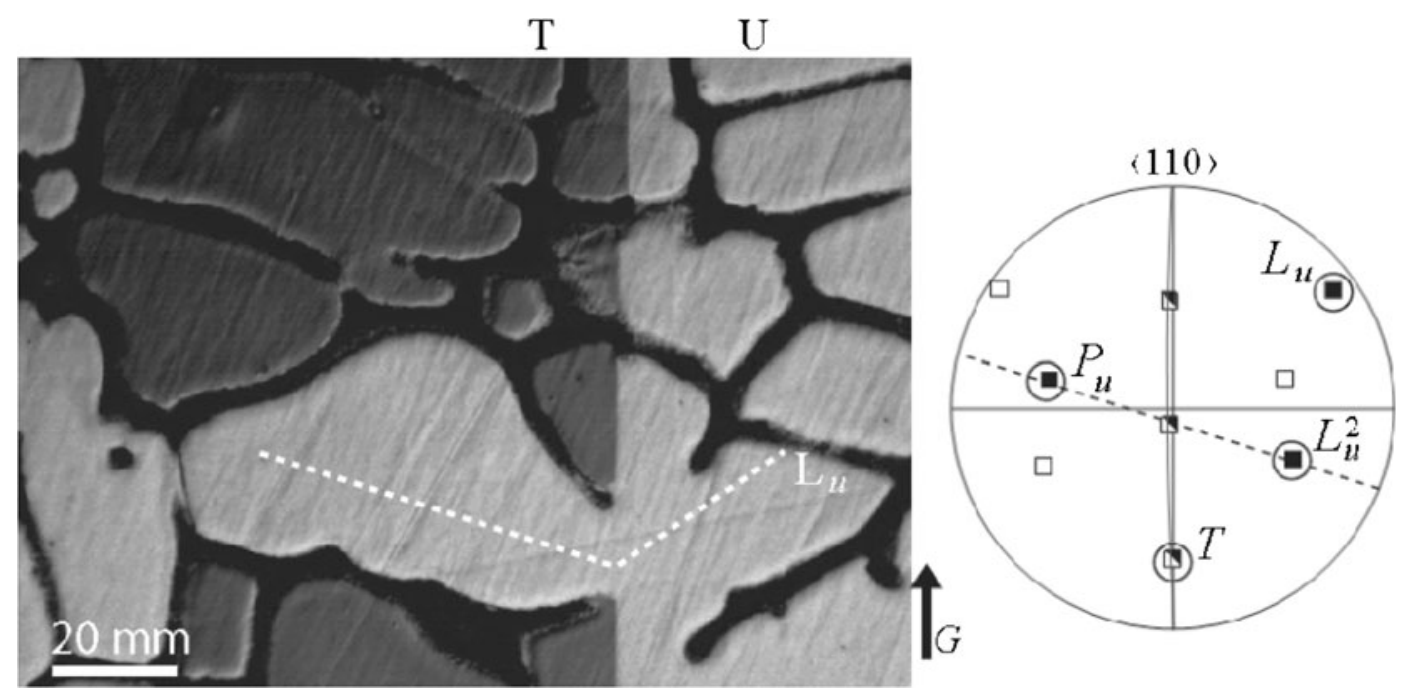

Fig. 3-Micrograph of a longitudinal section and corresponding $\langle 110\rangle$ pole figure showing the extension of a lateral $\langle 110\rangle$ arm of the untwinned region into the twinned region. 


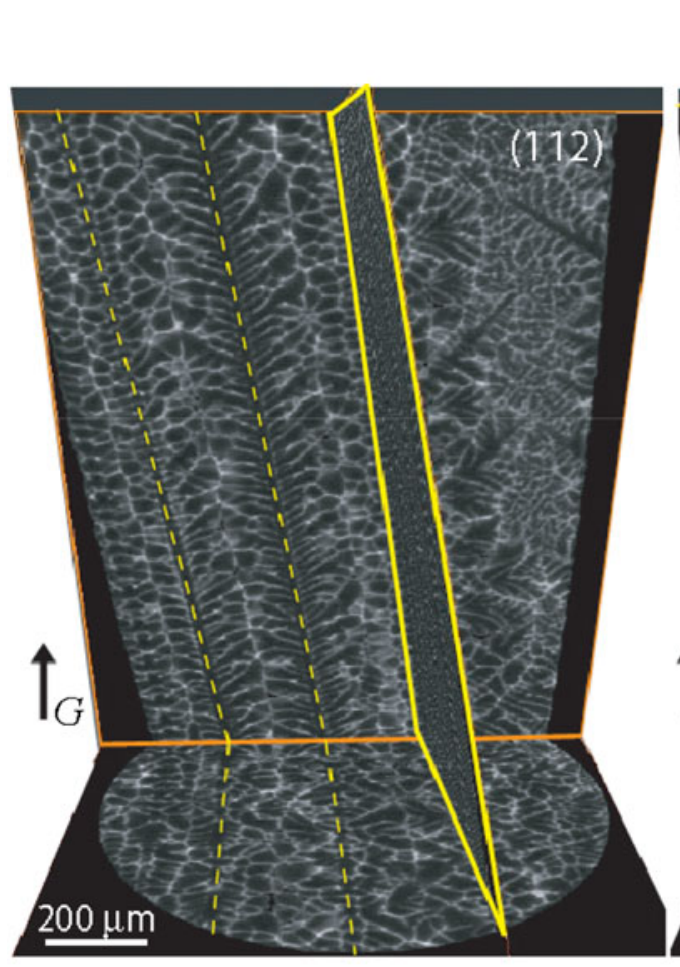

(a)

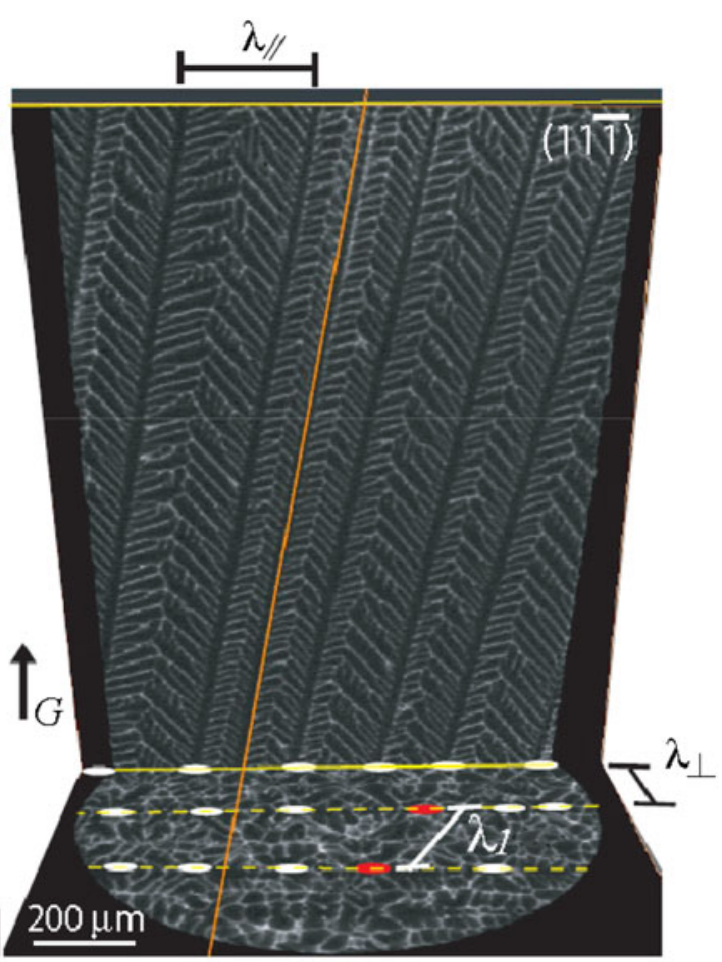

(b)

Fig. 4-2D-cuts of a 3D volume reconstructed by X-ray tomography microscopy for a quenched Al-26wt pct Zn specimen in a region close to the liquidus at the time of the quench: $(a)$ longitudinal section parallel to a (112) plane showing a continuous twinned dendrite trunk. The dashed and continuous yellow/grey lines indicate the traces of the twin planes; $(b)$ section parallel to a (11) twin plane containing the primary trunks. In both projections, the corresponding transverse section is shown, and in (b) the traces of the trunks have been indicated with small white ellipses together with the parameters $\lambda_{\perp}, \lambda_{/ /}$and $\lambda_{1}$.

the $(11 \overline{1})$ twin plane (highlighted with a continuous white line on the right). Two twinned dendrites can be identified in this section, and are indicated with white dashed lines. Analysis of this image has shown that the middle dendrite trunk is precisely parallel to the (112) plane shown in the figure, whereas the dendrite trunk on the left is slightly misoriented by a few degrees. As the trunk is also parallel to the (111) twin plane and is furthermore continuous in the (112) plane, this clearly shows that (1) The trunk grows exactly along a $\langle 110\rangle$ direction; and (2) The interruption of the coherent twin plane seen before in $\mathrm{Al}-\mathrm{Cu}$ alloys ${ }^{[7]}$ and also identified in Figure 3 does not occur through a dendrite trunk. This was confirmed by looking at similar sections of various volumes reconstructed along a vertical distance of a few millimeters in the same quenched specimen.

Figure 4(b) shows a longitudinal (11) section, i.e., parallel to the twin plane, of the same 3D reconstructed microstructure. The $\langle 110\rangle$ trunks and side arms growing parallel to this plane are clearly visible in this section. The small white ellipses in the transverse section at the bottom show the locations of several dendrite trunks. The spacing $\lambda_{\perp}$ is that from successive twin planes, while $\lambda_{/ /}$and $\lambda_{1}$ are the primary trunk spacings along the twin plane and in a transverse section, respectively. From our observations, it appears that (1) $\lambda_{1} \geq \lambda_{\perp}$; (2) $\lambda_{/ /}$can be larger or smaller than $\lambda_{\perp}$; (3) The selection of $\lambda_{1}$ and $\lambda_{/ 1}$ is similar to that of regular dendrites ${ }^{[20,21]}$ : when the trunk spacing becomes too small, a primary trunk is eliminated and when it is too large, a new twinned trunk might appear. Yet, there is a difference: A (coherent) twin plane is not translated and rarely eliminated during the growth process.

Other observations can be made through further analysis of Figure 4. First, it appears that there is a relatively stronger solute segregation in a direction perpendicular to the twin plane (Figure 4(a)), than in the direction parallel to the twin planes (Figure 4(b)). This is because $\langle 110\rangle$ secondary arms growing in this plane are twinned as well and adopt a doublon-type morphology, as shown by phase field simulations. ${ }^{[12,14]}$ Therefore, the associated microsegregation is reduced from the liquid pool being formed at their center. When these arms meet, they have also the same orientation (half split, twinned/untwinned) and thus can end solidification easily by coalescence (no coalescence undercooling $\left.{ }^{[22]}\right)$. On the contrary, microsegregation associated with $\langle 110\rangle$ - (or $\langle 100\rangle-$ ) arms growing away from the twin plane is similar to that of regular dendrites. Furthermore, they meet at incoherent twin boundaries and thus coalescence near the end of solidification is also more difficult. Second, it can be seen on the right of Figure 4(a) that in the large space of liquid left until the quench was applied, secondary and tertiary branches at $90 \mathrm{deg}$ from each other have formed. It seems that the presence of the twin plane imposes a preferred $\langle 110\rangle$ growth direction to trunks and secondary arms, but away from the twin plane, the system has a tendency to 
regain its regular growth directions which, for this zinc composition, ${ }^{[18]}$ correspond to $\langle 100\rangle$.

\section{Twin Growth Propagation}

The propagation of the dendritic network in a direction parallel to the twin plane is easy, as shown in the previous section. However, its extension in a direction perpendicular to the coherent planes requires the nucleation of a new stacking fault in a tertiary arm issued from secondary branches. ${ }^{[10]}$ While these stacking faults automatically give rise to twins during solidification, the alternated sequence of twinned and untwinned regions can only be respected if some preferential growth mechanisms occur afterward (on the basis of "what is finally seen is what has been selected"). Henry et $a .^{[10]}$ suggested that this was the role of melt convection. While this mechanism could possibly operate, the observations made in the previous section suggest another mechanism (see Figure 2). Indeed, one untwinned region can extend in a twinned part, as already shown in a transverse section of an Al$10 w t$ pet $\mathrm{Cu}$ specimen $^{[7]}$ and now in a longitudinal section of a quenched Al-Zn specimen (Figure 2). In order to clearly identify the branching mechanisms in a region where such extension occurs, Figure 5 shows a magnification of the region of the microstructure that contains $T_{2}$ and the upper part of $T_{3}$, just below the thermocouple location in Figure 2. In the bottom of the micrograph, a $\langle 100\rangle$ lateral side arm of the (light gray)

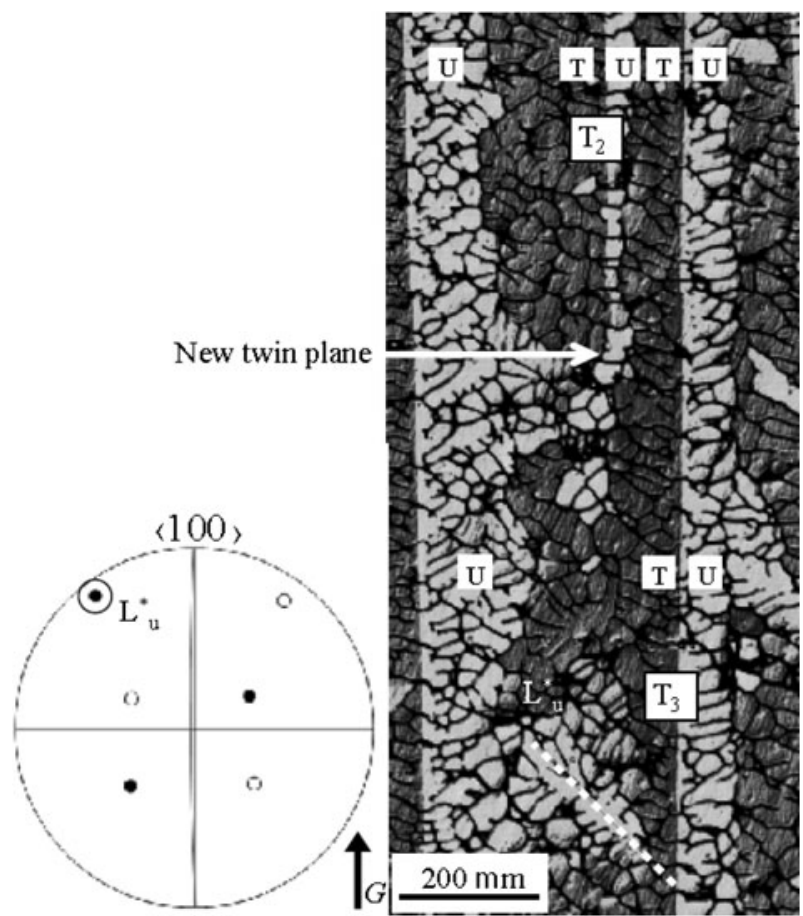

Fig. 5-Micrograph showing an extension of a $\langle 100\rangle$ arm of the untwinned region into the twinned region of the twinned dendrite labeled $T_{3}$ in Fig. 2. This extension gives eventually an origin to a new twin plane, labeled $T_{2}$. The alternance of untwinned (U) and twinned (T) lamellae is indicated in the micrograph. The $\langle 100\rangle$ direction of the arm growing at $45 \mathrm{deg}$ from the twin plane in the micrograph is indicated in the corresponding pole figure on the left. untwinned region of $T_{3}$ clearly extends into the (dark) twinned lamella. Its growth direction is indicated in the $\langle 100\rangle$ pole figure on the left. Further up along the direction of $G$, a new pair of lamellae, labeled $T_{2}$, appears on the left of $T_{3}$. Initially, the boundary between these two newly formed lamellae does not appear to be a coherent twin, but as growth proceeds, it becomes straight and coherent.

This propagation mechanism is further clarified in the schematic drawing of Figure 6. The top figure shows two twin dendrites seen from the top (i.e., from the liquid side), the dark and light gray shades representing the untwinned and twinned parts of the microstructure, respectively. In the twin plane, the $\langle 110\rangle$ trunk is shown with a circle, while the two other $\langle 110\rangle$ arms growing parallel to this plane at $90 \mathrm{deg}$ on each side of the trunk are indicated by ellipses. For each side of a twinned

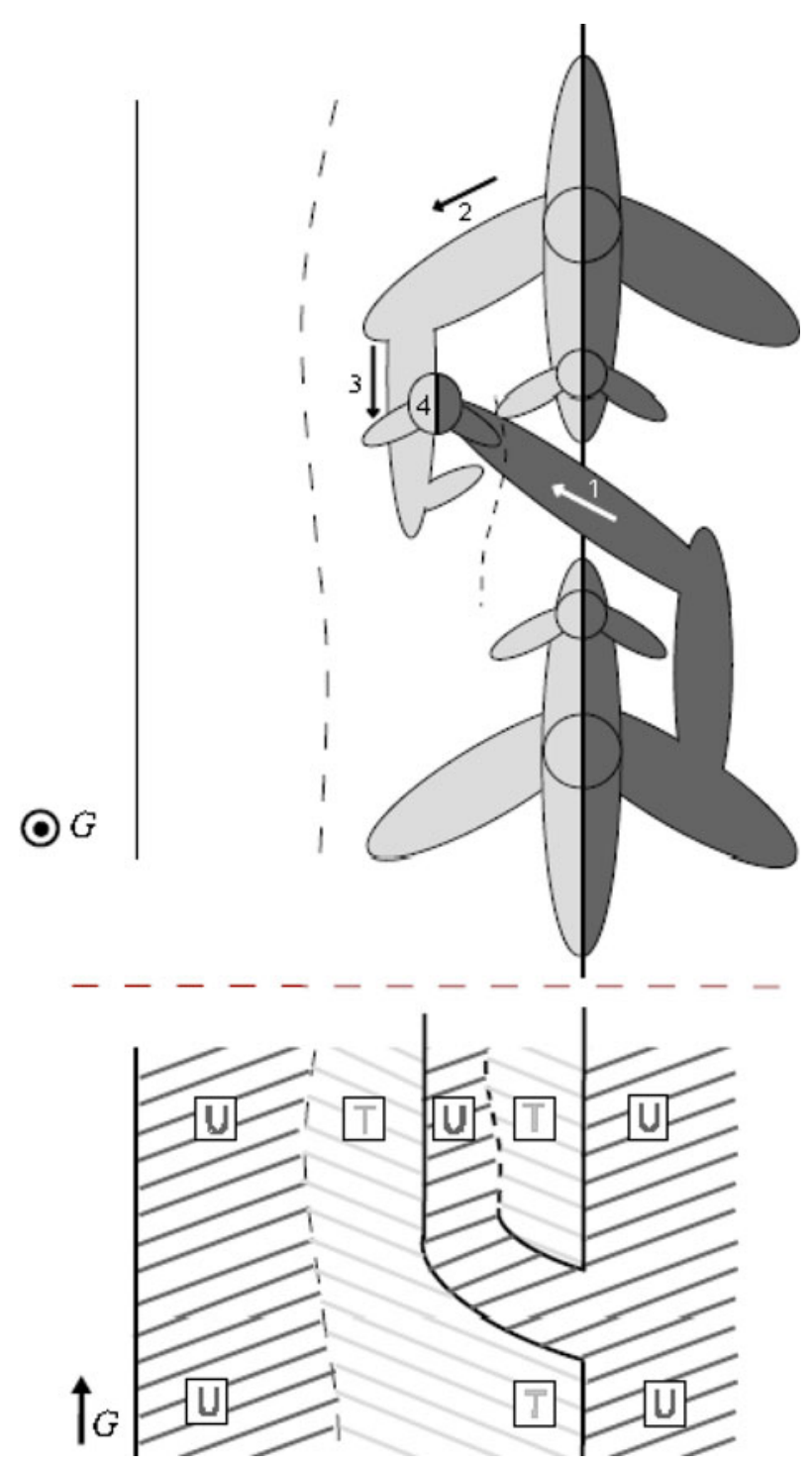

Fig. 6-Schematic representation of the twin lateral propagation mechanism. In the longitudinal section, parallel to $G$ (bottom), only the lamellar sequence is drawn. In the transverse section, perpendicular to $G$ (top), a detailed schematic sequence of twin multiplication is described (see text). 
dendrite, there are three other $\langle 110\rangle$ directions: two of them, also represented by ellipses, are at $60 \mathrm{deg}$ on each side of the trunk, but in another (111) plane, while the third one is at $90 \mathrm{deg}$ (not represented here). In order to explain the formation of a new twin boundary, e.g., $T_{2}$ in Figure 5, we propose the following mechanism:

(a) A $\langle 110\rangle$ (or sometimes $\langle 100\rangle$ ) lateral arm of the untwinned dendrite (labeled 1 in this figure) grows into the twinned region. To do so, it has to compete with the other dendrite arms. As for the growth competition that occurs between secondary and tertiary arms of regular $\langle 100\rangle$ dendrites, ${ }^{[23-25]}$ this occurs via solutal interactions and strongly depends on the orientation of the thermal gradient with respect to the structure. Unlike observations of this competition for $\langle 100\rangle$ dendrites which, for organic analogs, can be viewed in a (100) plane, the situation here is fully $3 \mathrm{D}$.

(b) A $\langle 110\rangle$ lateral arm (labeled 2 in Figure 6) of the twinned part grows and starts forming tertiary branches as well (labeled 3 in this case).

(c) The untwinned arm 1 and twinned arm 3 can meet in between the two original twin coherent planes, and provided the spacing is large enough, a new twinned trunk (labeled 4) can form and grow.

Although Figure 6 shows the formation of a new twinned primary trunk form a quaternary untwinned arm and a tertiary twinned arm, a similar mechanism can occur with a multiplicity of other $\langle 110\rangle$ (or $\langle 100\rangle$ ) arms. This mechanism has been put forward to explain the formation of a new coherent twin plane in between two existing ones (in this case $T_{2}$ formed between $T_{1}$ and $T_{3}$ in Figure 5): it is perfectly compatible with the fact that the two initially growing planes slightly diverge (as is the case for feathery grain structures ${ }^{[10]}$ ), and thus see their spacings increase during growth. At some point, secondary arms have enough place to grow and form a new twinned primary trunk. The same mechanism can occur at the boundary of a twinned grain with other (regular) grains, thus extending the twinned structure in a direction perpendicular to the twin planes, at the expense of regular dendritic grains. This is an alternative to the spontaneous stacking fault formation mechanism suggested by Henry et al.. ${ }^{[0]}$

\section{Competition Mechanisms}

While the competition of regular dendrites and the formation of solidification textures are well known since the work of Chalmers, ${ }^{[25-27]}$ the growth advantage that twinned aluminum dendrites can have over regular ones under certain conditions remains unclear. The weak anisotropy of aluminum, ${ }^{[28]}$ the reduced solute segregation associated with a doublon-type twinned dendrite tip, ${ }^{[14-16]}$ and the solidification conditions (high thermal gradient, presence of convection, etc.) all play an important role.

Figure 7 illustrates, if not explains, the growth advantage of a twinned $\langle 110\rangle$ dendrite over a regular $\langle 100\rangle$ one. It was obtained from the same quenched specimen presented in Figure 2. Near the liquidus

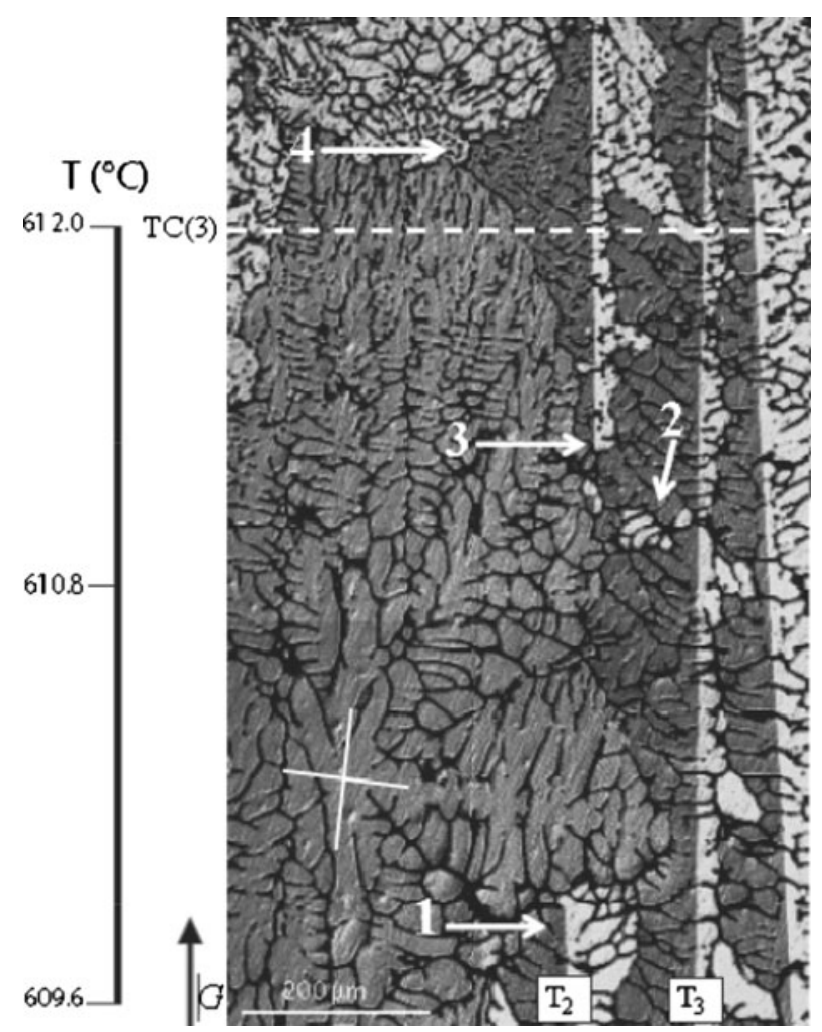

Fig. 7-Competition between regular $\langle 100\rangle$ and twinned $\langle 110\rangle$ dendrites in a quenched Al-26 wt pet $\mathrm{Zn}$ specimen: (1) Twinned dendrite overgrown by an equiaxed grain nucleated ahead of the columnar front; (2) Lateral propagation of a twin plane; (3) Twin plane appearing next to the columnar $\langle 110\rangle$ structure; and (4) regular $\langle 100\rangle$ trunks overgrown by lateral arms of a twinned dendrite.

isotherm (dashed line), a $\langle 100\rangle$ equiaxed grain can be seen on the left of the micrograph. Most likely, it has nucleated ahead of the twinned columnar front during solidification and not during the quench, as can be evidenced from its secondary dendrite arms which have about the same spacing as those of the neighbor twinned dendrite $T_{3}$ (at the same height). As a matter of fact, this grain has already stopped the growth of the trunk $T_{1}$ (see Figure 2). In this section, it has developed four main arms along orthogonal $\langle 100\rangle$ directions indicated by the small cross at the center. The two arms growing perpendicularly to the thermal gradient naturally encounters the arms of the neighbor twin dendrites $T_{3}$ and have stopped the growth of the trunk $T_{2}$ (see location " 1 " in Figure 7). During further directional solidification (and possibly quench), this equiaxed grain evolves into a rather columnar structure that competes with the other twinned dendrites. After a few hundred microns, either a new twin plane forms from the dendrite $T_{3}$ (see location " 2 " in Figure 7), by the same mechanism described before, or the twin dendrite $T_{2}$ reappears from a region located above or below this section (see location "3" in Figure 7). The lateral arms of this twinned dendrite start to overgrow the primary trunks of the $\langle 100\rangle$ grain (see location "4" in Figure 7), before an equiaxed grain forms in the quenched region (top of the figure). The occurrence of these phenomena shows that (1) Equiaxed grains can form ahead of 
twinned columnar dendrites, thereby blocking their trunks as in the well-known columnar-to-equiaxed transition; (2) In a transient regime, both "equiaxed" $\langle 100\rangle$ and twinned $\langle 110\rangle$ dendrites compete via solutal interactions between side arms; and (3) As the side arms of twinned dendrites are inclined at $60 \mathrm{deg}$ from the trunk, they probably have a growth advantage over those of regular dendrites which are growing perpendicularly to the thermal gradient. Overall, once they form, twinned dendrites seem to have a definite growth advantage over regular dendrites.

\section{CONCLUSIONS}

A modified DS installation with the ability to quench twinned dendrites during growth has been successfully developed, which has enabled new characterization of twinned dendrite morphology. In particular, this apparatus has produced specimens with very good X-ray contrast for subsequent X-ray tomography microscopy analysis. Observations made on the resulting 3D-reconstructed images have confirmed previous 2D observations of twinned dendrites including complex branch morphology, asymmetric nature of the solute distribution profile, and the adaptive and fairly isotropic distribution of primary trunks in an Al-Zn alloy system. By correlating the 3D observations with those performed in regular DS specimens, the following conclusions can be drawn:

1. Frequently, one side of a twinned dendrite extends into the other side, interrupting locally the coherency of the twin plane at the scale of the lamellae (not of the trunks themselves). These extensions occur only at the level of secondary or higher order branches.

2. The extension of a feathery grain in a direction perpendicular to the twin plane does not necessarily require the nucleation of a stacking fault, as suggested by Henry et al. ${ }^{[10]}$ An alternative mechanism is the extension of side arms of a single twinned dendrite on the other side of the coherent twin plane. In a similar fashion as the growth of regular dendrites, this extension of higher-order side arms can therefore self-propagate a twinned dendrite.

3. The clear growth advantage of twinned dendrites is due to the orientation of $\langle 110\rangle$ (or $\langle 100\rangle$ ) side arms of twinned dendrites at $60 \mathrm{deg}$ (respectively $45 \mathrm{deg}$ ) from $\langle 110\rangle$ twinned trunks, their increase in number with respect to regular dendrites, and their orientation with respect to $G$.

\section{ACKNOWLEDGMENTS}

The authors would like to thank the staff of the Interdisciplinary Center for Electron Microscopy of the Ecole Polytechnique Fédérale de Lausanne (EPFL), and in particular, Dr Emmanuelle Boehm, for the help in the EBSD measurements. The help of Jean-Daniel Wagnière in constructing the quenching device is also greatly appreciated. The authors would also like to thank Prof. M. Stampanoni and Dr. Samuel McDonald for their help in conducting the tomography experiments using the TOMCAT beamline at the SLS.

\section{REFERENCES}

1. J. Herenguel: Rev. Metall., 1948, vol. 45 (5), pp. 339-46.

2. R.E. Spear, R.T. Craig, and C.R. Howle: J. Met., 1971, vol. 23, p. 42.

3. W. Schneider. 4th International Summer School on Al Alloy Technology, Trondheim, Norway, 1997.

4. S. Henry, G. Gruen, and M. Rappaz: Metall. Mater. Trans. A, 2004, vol. 35A, pp. 2495-01.

5. A.N. Turchin, D.G. Eskin, and L. Katgerman: Metall. Mater. Trans. A, 2007, vol. 38A, pp. 1317-29.

6. S. Henry, T. Minghetti, and M. Rappaz: Acta Mater., 1998, vol. 46 (18), pp. 2495-01.

7. M.A. Salgado-Ordorica and M. Rappaz: Acta Mater., 2008, vol. 56, pp. 5708-18.

8. R.L. Morris, J.R. Carruthers, A. Plumtree, and W.C. Winegard: AIME Metall. Soc. Trans., 1966, vol. 236 (9), pp. 1286-91.

9. J. Herenguel: Rev. Metall., 1949, vol. 46 (5), pp. 309-14.

10. S. Henry, P. Jarry, and M. Rappaz: Metall. Mater. Trans. A, 1998, vol. 29A, pp. 2807-17.

11. S. Henry: PhD Thesis, Ecole Polytechnique Fédérale de Lausanne, 1999.

12. M.A. Salgado-Ordorica, J.-L. Desbiolles, and M. Rappaz: MCW ASP XII Proceedings, by S. L. Cockcroft and D. Maijer, XII, 2008, pp. 545-552.

13. M. Rappaz, J. Friedli, A. Mariaux, and M.-A. Salgado-Ordorica: Scripta Mater., 2010, vol. 62, pp. 904-09.

14. M.A. Salgado-Ordorica, J.-L. Desbiolles, and M. Rappaz: Acta Mater., 2011, vol. 50 (13), pp. 5074-84.

15. M.A. Salgado-Ordorica, P. Burdet, M. Cantoni, and M. Rappaz: Acta Mater., 2011, vol. 50 (13), pp. 5085-91.

16. M.A. Salgado-Ordorica, J. Valloton, and M. Rappaz: Scripta Mater., 2009, vol. 61, pp. 367-70.

17. H. Anada, K. Funaki, Y. Nakashima, H. Sawabu, and E. Tada: J. Jpn. Inst. Light Met., 1986, vol. 36 (9), pp. 562-70.

18. F. Gonzales and M. Rappaz: Metall. Mater. Trans. A, 2006, vol. 37A, p. 2797.

19. W. Kurz, B. Giovanola, and R. Trivedi: Acta Metall., 1986, vol. 34 (5), pp. 823-30.

20. J.T. Mason, J.D. Verhoeven, and R. Trivedi: J. Cryst. Growth, 1982, vol. 59 (3), pp. 516-24.

21. J.A. Warren and J.S. Langer: Phylos. Rev. E, 1993, vol. 47 (4), pp. 2702-12.

22. M. Rappaz, A. Jacot, and W.J. Boettinger: Metall. Mater. Trans. A, 2003, vol. 34A, pp. 467-79.

23. H. Esaka: PhD Thesis, Ecole Polytechnique Fédérale de Lausanne, 1986.

24. H. Esaka, W. Kurz, and R. Trivedi: Solidification Processes, The Institute of Metals, London, 1988.

25. Ch.-A. Gandin, M. Eshelman, and R. Trivedi: Metall. Mater. Trans. A, 1996, vol. 27A, pp. 2727-37.

26. D. Walton and B. Chalmers: Trans. AIME, 1959, vol. 215, pp. $847-55$.

27. C.A. Gandin, M. Rappaz, D. West, and B.L. Adams: Metall. Mater. Trans. A, 1995, vol. 26A, pp. 1543-51.

28. R.E. Napolitano and S. Liu: Phylos. Rev. B, 2004, vol. 70, p. 214103. 